\title{
A Determinantal Proof of the Product Formula for the Multivariate Transfinite Diameter
}

\author{
by \\ Jean-Paul CALVI and PHUNG VAN MANH
}

Presented by Józef SICIAK

Summary. We give an elementary proof of the product formula for the multivariate transfinite diameter using multivariate Leja sequences and an identity on vandermondians.

1. Introduction and statement of the result. Let $\mathbb{N}_{d}^{n}$ denote the set of $n$-indices of length at most $d$ endowed with the graded lexicographic order $(\prec)$. The cardinality of $\mathbb{N}_{d}^{n}$, denoted by $N_{d}^{n}$, is equal to $\left(\begin{array}{c}n+d \\ d\end{array}\right)$. A vandermondian (of order $d$ ) is the determinant of an $N_{d}^{n} \times N_{d}^{n}$ matrix of the form $\left(z_{\alpha}^{\beta}\right)$ where $z_{\alpha} \in \mathbb{C}^{n},[\cdot]^{\beta}$ is the usual monomial and the rows and columns are ordered according to $\prec$. Such a determinant is denoted by $\operatorname{VDM}(\mathbf{z})$ where $\mathbf{z}:=\left(z_{\alpha}: \alpha \in \mathbb{N}_{d}^{n}\right)$. It is a polynomial of degree

$$
\ell_{d}^{n}:=n\left(\begin{array}{c}
n+d \\
n+1
\end{array}\right)
$$

in the $\left(N_{d}^{n}\right)^{n}$ coordinates of the $z_{\alpha}$ 's. The $d$ th diameter $D_{d}(K)$ of a compact subset $K$ of $\mathbb{C}^{n}$ is defined by

$$
D_{d}(K)=\sup \left\{|\operatorname{VDM}(\mathbf{z})|^{1 / \ell_{d}^{n}}: \mathbf{z} \in K^{N_{d}^{n}}\right\},
$$

and a collection $\mathbf{z}$ for which the supremum is achieved in (1) is called a Fekete system (of order $d$ ) for $K$. Now, the transfinite diameter $D(K)$ is the limit of $D_{d}(K)$ as $d$ goes to $\infty$. That such a limit exists is by no means obvious (when $n>1$ ). It is a beautiful result of $\mathrm{V}$. Zaharjuta [9] who not only proved the convergence of $\left(D_{d}(K)\right)$ but also related its limit to complex polynomial approximation.

2000 Mathematics Subject Classification: Primary 32U20.

Key words and phrases: multivariate transfinite diameter, Vandermonde determinants, Leja sequences. 
Here, for the sake of brevity, we shall content ourselves with recalling one useful relation. Given $\alpha \in \mathbb{N}^{n}$ we define

$$
T(\alpha, K):=\inf \left\{\|\tau\|_{K}: \tau \in \mathbb{U P}_{\alpha}\right\}
$$

where $\mathbb{U P}_{\alpha}$ is the set of polynomials with $\prec$-leading monomial $z^{\alpha}$, i.e., $\tau(z)=$ $z^{\alpha}+\sum_{\beta \prec \alpha} c_{\beta} z^{\beta}$ and $\|\cdot\|_{K}$ is the sup-norm on $K$. A polynomial for which the infimum is achieved is called an $\alpha$-Chebyshev polynomial. Zaharjuta showed that

$$
D(K)=\lim _{d \rightarrow \infty}\left(\prod_{|\alpha|=d} T(\alpha, K)\right)^{1 /\left(d h_{d}^{n}\right)},
$$

where

$$
h_{d}^{n}:=\left(\begin{array}{c}
n+d-1 \\
d
\end{array}\right)=\left|\mathbb{N}_{d}^{n} \backslash \mathbb{N}_{d-1}^{n}\right| .
$$

Just like its (better known) univariate analogue, the multivariate transfinite diameter is intimately connected with (pluri)potential theory. Most of what is known so far about these relations can be found in $[4,5,2]$ and in the references of those papers. Our note rather deals with the elementary properties of the transfinite diameter and gives a (mainly) algebraic proof of the following formula.

THEOREM 1. For $i=1,2$, let $K_{i}$ be a compact subset of $\mathbb{C}^{n_{i}}, n=n_{1}+n_{2}$ and $K:=K_{1} \times K_{2} \subset \mathbb{C}^{n}$. Then

$$
D(K)=\left(D\left(K_{1}\right)\right)^{n_{1} / n} \cdot\left(D\left(K_{2}\right)\right)^{n_{2} / n} .
$$

This result was first proved in [4, pp. 286-292] by computing transfinite diameters with the help of orthogonal polynomials with respect to a positive measure satisfying the Bernstein-Markov inequality. That proof requires two non-immediate facts. First, that the map $K \mapsto D(K)$ is continuous under non-increasing sequences (of compact sets) and, second, that, for every $K$, there exists a measure satisfying the Bernstein-Markov inequality with support as close as we like to $K$.

However, seeing the relatively simple formula (3), we may suspect there does exist a proof that works with the determinants involved in the definition (1) of the $d$ th diameters. Actually such a proof was found a long time ago by Schiffer and Siciak [8] but it worked only in the case $n_{i}=1$. The proof presented here is much in the same spirit as theirs. The idea is to take a Fekete system for $K_{1}$, another for $K_{2}$ and to "combine" them to form something like a Fekete system for $K_{1} \times K_{2}$. We shall amend this very rough idea and elaborate on it in the next section, and our proof of (a slightly more informative version of) Theorem 1 will easily follow in the last section. 
2. Tools. (a) We shall not be able to work with Fekete systems but rather with similar extremal collections of points, called (block) Leja sequences. If $E$ is a compact subset of $\mathbb{C}^{m}$, points of such a sequence are indexed by $m$-indices and are inductively constructed as follows. We choose any $a_{0} \in E$ and if $\mathcal{L}_{d}:=\left(a_{\alpha}:|\alpha| \leq d\right)$ then we define $\mathcal{L}_{d+1}=\left(a_{\alpha}:|\alpha| \leq d+1\right)$ by requiring

$$
\operatorname{vDM}\left(\mathcal{L}_{d+1}\right)=\sup \left\{|\operatorname{VDM}(\mathbf{z})|: \mathbf{z} \in E^{N_{d+1}^{m}}, z_{\alpha}=a_{\alpha} \text { for }|\alpha| \leq d\right\} .
$$

Note that it is not excluded that $\operatorname{vDM}\left(\mathcal{L}_{d}\right)=0$ for every $d$. A very similar sequence, call it a (point) Leja sequence, was introduced independently by Jędrzejowski [7] and Bloom et al. [3]. In both cases, the authors constructed it by adding one point at each step (so that they needed $N_{d}^{m}$ steps to construct $N_{d}^{m}$ points) whereas we add a whole block of $h_{d}^{m}$ points at step $d$ (so that we need $d+1$ steps to construct $N_{d}^{m}$ points). We could work as well with their (point) Leja sequences, but since the notion of block is essential in our discussion, as will soon be apparent, we prefer to stick to the more natural setting. The point is that, as long as we are concerned with transfinite diameters, (block) Leja sequences behave like sequences of Fekete systems.

Theorem 2. We have

$$
D(E)=\lim _{d \rightarrow \infty}\left|\operatorname{VDM}\left(\mathcal{L}_{d}\right)\right|^{1 / \ell_{d}^{m}}
$$

Proof. We only sketch it for it is a mere adaptation of the proof given in [7] or in [3, pp. 461-462] for a (point) Leja sequence. First, since, by definition, the Fekete system is the best possible choice, we have

$$
D(E) \geq \limsup _{d \rightarrow \infty}\left|\operatorname{VDM}\left(\mathcal{L}_{d}\right)\right|^{1 / \ell_{d}^{m}} .
$$

Hence we just need to prove that the lim inf satisfies the reverse inequality. We may also assume that $E$ is unisolvent for $\mathbb{P}\left(\mathbb{C}^{m}\right)$ (that is, no non-zero polynomial vanishes identically on $E$ ) for otherwise the conclusion is easy (see the argument in [7, p. 69]). We shall prove by induction that

$$
\left|\operatorname{vDM}\left(\mathcal{L}_{d}\right)\right| \geq \prod_{|\alpha| \leq d} T(\alpha, E) \quad(d \geq 0)
$$

and a (careful) use of (2) will then yield the required lim inf inequality (see $[7,3]$ for details regarding this last step).

The inequality (5) is obvious for $d=0$. We assume it holds true for $d$ and proceed to prove it for $d+1$. Let $\mathbf{z}=\left(z_{\alpha}\right)$ as in (4). For every $\beta$ of length $d+1$, we put

$$
M_{\beta}:=\operatorname{det}\left(z_{u}^{v}: u \preceq \beta, v \preceq \beta\right) .
$$

Since, the $\prec$-first $N_{d}^{m}$ entries of $\mathbf{z}$ are fixed (equal to the $a_{\alpha}$ 's), $M_{\beta}$ is a (continuous) function of the variables $z_{u}$ for $d<|u|, u \preceq \beta$. Let $\gamma$ be the $\prec$-smallest $m$-index of length $d+1$. Expanding $M_{\gamma}$ with respect to the last 
column, we see that $M_{\gamma}=\operatorname{vDM}\left(\mathcal{L}_{d}\right) \tau_{\gamma}\left(z_{\gamma}\right)$ where $\tau_{\gamma} \in \mathbb{U P}_{\gamma}$ and the coefficients of $\tau_{\gamma}$ depend only on the $a_{\alpha}$ 's. Such a formula is valid however only because $\operatorname{vDM}\left(\mathcal{L}_{d}\right) \neq 0$ and this follows from the induction hypothesis. Indeed, since $E$ is unisolvent, $T(\alpha, E)>0$ for every $\alpha$, which, by (5), ensures $\operatorname{VDM}\left(\mathcal{L}_{d}\right) \neq 0$. Now, using again the unisolvence of $E$, we may choose (and fix) $z_{\gamma} \in E$ so that $\left|\tau_{\gamma}\left(z_{\gamma}\right)\right|=\left\|\tau_{\gamma}\right\|_{E}>0$ (which implies $M_{\gamma} \neq 0$ ). Let now $\theta$ be the $\prec$-successor of $\gamma$. Expanding $M_{\theta}$ with respect to its last column we find $M_{\theta}=M_{\gamma} \tau_{\theta}\left(z_{\theta}\right)$ where $\tau_{\theta} \in \mathbb{U P}_{\theta}$ and the coefficients of $\tau_{\theta}$ depend only on the $a_{\alpha}$ 's and on the already fixed $z_{\gamma}$. Again, this is possible because $M_{\gamma} \neq 0$. Using the unisolvence of $E$, we may next find (and fix) $z_{\theta} \in E$ such that $\left|\tau_{\theta}\left(z_{\theta}\right)\right|=\left\|\tau_{\theta}\right\|_{E}>0$ (which implies $M_{\theta} \neq 0$ ). At this point, we have

$$
\left|M_{\theta}\right|=\left|\operatorname{VDM}\left(\mathcal{L}_{d}\right)\right|\left\|\tau_{\gamma}\right\|_{E}\left\|\tau_{\theta}\right\|_{E} \geq\left|\operatorname{VDM}\left(\mathcal{L}_{d}\right)\right| T(\gamma, E) T(\theta, E) .
$$

Continuing in this way, after $h_{d+1}^{m}$ inductive steps, for every $\alpha$ of length $d+1$, we construct a polynomial $\tau_{\alpha} \in \mathbb{U P}_{\alpha}$ and $z_{\alpha} \in E$ with $\left\|\tau_{\alpha}\right\|_{E}=\left|\tau_{\alpha}\left(z_{\alpha}\right)\right|>0$ such that, for the corresponding system $\mathbf{z}$, we have

$$
\operatorname{VDM}(\mathbf{z})=\operatorname{VDM}\left(\mathcal{L}_{d}\right) \prod_{|\alpha|=d+1} \tau_{\alpha}\left(z_{\alpha}\right)
$$

hence $|\operatorname{VDm}(\mathbf{z})| \geq\left|\operatorname{VDm}\left(\mathcal{L}_{d}\right)\right| \prod_{|\alpha|=d+1} T(\alpha, E)$. By construction of $\mathcal{L}_{d+1}$, we therefore have $\left|\operatorname{VDm}\left(\mathcal{L}_{d+1}\right)\right| \geq\left|\operatorname{vDM}\left(\mathcal{L}_{d}\right)\right| \prod_{|\alpha|=d+1} T(\alpha, E)$. Now, using again the induction hypothesis we arrive at $\operatorname{vDM}\left(\mathcal{L}_{d+1}\right) \geq \prod_{|\alpha| \leq d+1} T(\alpha, E)$, which is (5) for $d+1$.

The interested reader should consult the paper [1] for an application of (point) Leja sequences to the reconstruction of the pluricomplex Green function.

(b) Following our idea, we want to combine Leja sequences for $K_{1}$ and $K_{2}$ to get something like a Leja sequence for $K_{1} \times K_{2}$. We first say what we mean by combining sequences. We shall use a (natural) process introduced in [6] which generalizes an old idea of Biermann. Given, for $i=1,2$, a collection $X_{i}=\left(x_{i, \alpha}:|\alpha| \leq d\right)$ of $N_{d}^{n_{i}}$ points in $\mathbb{C}^{n_{i}}$, the intertwining of $X_{1}$ and $X_{2}$ is the collection of $N_{d}^{n_{1}+n_{2}}$ points in $\mathbb{C}^{n_{1}+n_{2}}$ defined by $X_{1} \oplus X_{2}:=$ $\left(\left(x_{1, \alpha}, x_{2, \beta}\right):|\alpha|+|\beta| \leq d\right)$. The following theorem is proved in [6, Sec. 5] by using multivariate Lagrange interpolation theory. It is this result which enables us to show that the intertwining of Leja sequences for the factor sets $K_{i}$ behaves like a Leja sequence for the product set $K=K_{1} \times K_{2}$. It was found and used by Schiffer and Siciak in the case $n_{i}=1$ for which the intertwining of the $X_{i}$ reduces to the aforementioned construction of Biermann. 
Theorem 3. Let $X_{i}, i=1,2$, and $X_{1} \oplus X_{2}$ as above. Then

$$
\operatorname{VDM}\left(X_{1} \oplus X_{2}\right)=\prod_{j=1}^{d}\left(\operatorname{vDM}\left(X_{1}^{j}\right)\right)^{h_{d-j}^{n_{2}}} \prod_{j=1}^{d}\left(\operatorname{VDM}\left(X_{2}^{j}\right)\right)^{h_{d-j}^{n_{1}},}
$$

where $X_{i}^{j}$ is the $j$ th block of $X_{i}$, that is, $X_{i}^{j}:=\left(x_{i, \alpha}:|\alpha| \leq j\right)$.

It is very important for us that for every $j, 1 \leq j \leq d$, the $j$ th block of the $d$ th term $\mathcal{L}_{i, d}$ of the Leja sequence for $K_{i}$ is just the $j$ th term of this same sequence, i.e., $\mathcal{L}_{i, d}^{j}=\mathcal{L}_{i, j}$. It has been constructed precisely in order that this property be satisfied. The same would no longer be true with Fekete systems and this is the very reason why we need to use Leja sequences here. This being said, we immediately deduce the following corollary to Theorem 3 .

Corollary 4. For $i=1,2$, let $\left(\mathcal{L}_{i, d}\right)$ be a Leja sequence for $K_{i}$. Then

$$
\operatorname{VDM}\left(\mathcal{L}_{1, d} \oplus \mathcal{L}_{2, d}\right)=\prod_{j=1}^{d}\left(\operatorname{vDM}\left(\mathcal{L}_{1, j}\right)\right)^{h_{d-j}^{n_{2}}} \prod_{j=1}^{d}\left(\operatorname{VDM}\left(\mathcal{L}_{2, j}\right)\right)^{h_{d-j}^{n_{1}}}
$$

(c) We finally need some information to be able to calculate with the fairly complicated exponents involved in the various formulae.

Lemma 5. Let $d, n_{i}, i=1,2$, be positive integers and $n=n_{1}+n_{2}$. Then

$$
\sum_{k=1}^{d} k h_{k}^{n_{1}} h_{d-k}^{n_{2}}=\left(n_{1} / n\right) d h_{d}^{n}
$$

Proof. This is Lemma 3 in [4]. It is proved using a standard generating power series technique.

Lemma 6. Let $d, n_{i}, i=1,2$, be positive integers and $n=n_{1}+n_{2}$. Then

$$
\sum_{k=1}^{d} \ell_{k}^{n_{1}} h_{d-k}^{n_{2}}=\left(n_{1} / n\right) \ell_{d}^{n}
$$

Proof. Lemma 5 (applied with $n_{1}+1$ ) yields

$$
\sum_{k=1}^{d} k h_{k}^{n_{1}+1} h_{d-k}^{n_{2}}=\frac{n_{1}+1}{n+1} d h_{d}^{n+1}
$$

and the claim follows by observing that, for every $m>0$,

$$
m h_{m}^{n_{1}+1}=\frac{n_{1}+1}{n_{1}} \ell_{m}^{n_{1}}
$$

3. Abridged proof of Theorem 1. As a by-product of our proof, we shall see that the intertwining of Leja sequences for $K_{1}$ and $K_{2}$ permits one to recover the transfinite diameter of $K=K_{1} \times K_{2}$. Note that it is very 
unlikely that this intertwining sequence itself is a Leja sequence for $K$. We continue to denote by $\mathcal{L}_{i, d}$ a (block) Leja sequence for $K_{i}$.

STEP 1 . We prove $(D(K))^{n} \geq\left(D\left(K_{1}\right)\right)^{n_{1}} \cdot\left(D\left(K_{2}\right)\right)^{n_{2}}$. Since $D_{d}(K) \geq$ $\left|\operatorname{vDM}\left(\mathcal{L}_{1, d} \oplus \mathcal{L}_{2, d}\right)\right|^{1 / \ell_{d}^{n}}$, the inequality follows from the next lemma.

Lemma 7. We have

$$
\lim _{d \rightarrow \infty}\left|\operatorname{VDM}\left(\mathcal{L}_{1, d} \oplus \mathcal{L}_{2, d}\right)\right|^{1 / \ell_{d}^{n}}=\left(D\left(K_{1}\right)\right)^{n_{1} / n} \cdot\left(D\left(K_{2}\right)\right)^{n_{2} / n} .
$$

Proof. The details are a bit tedious but standard. We shall assume $D\left(K_{i}\right)$ $>0$. The proof is even simpler if one of the $D\left(K_{i}\right)$ is 0 . Fix $\varepsilon>0$. In view of Theorem 2 for $d$ large enough, say $d \geq d_{0}$, we have

$$
e^{-\varepsilon} D\left(K_{i}\right) \leq\left|\operatorname{VDM}\left(\mathcal{L}_{i, d}\right)\right|^{1 / \ell_{d}^{n_{i}}} \leq e^{\varepsilon} D\left(K_{i}\right) .
$$

Choose positive $r<R$ (depending on $\varepsilon$ ) such that

$$
r D\left(K_{i}\right) \leq\left|\operatorname{VDM}\left(\mathcal{L}_{i, d}\right)\right|^{1 / \ell_{d}^{n_{i}}} \leq R D\left(K_{i}\right) \quad \text { for } d<d_{0} .
$$

(Here we need $\operatorname{vDm}\left(\mathcal{L}_{i, d}\right) \neq 0$. This follows from (5) which holds true since $D\left(K_{i}\right)>0$ implies that $K_{i}$ is unisolvent for $\mathbb{P}_{d}\left(\mathbb{C}^{n}\right)$.) Working with $i=1$ and writing

$$
\left(\prod_{j=1}^{d}\left(\operatorname{vDM}\left(\mathcal{L}_{1, j}\right)\right)^{h_{d-j}^{n_{2}}}\right)^{1 / \ell_{d}^{n}}=\prod_{j=1}^{d}\left(\left|\operatorname{vDM}\left(\mathcal{L}_{1, j}\right)\right|^{1 / \ell_{j}^{n_{1}}}\right)^{\ell_{j}^{n_{1}} h_{d-j}^{n_{2}} / \ell_{d}^{n}},
$$

we readily infer, on distinguishing the cases $d \geq d_{0}$ and $d<d_{0}$ and using (8), (9) and Lemma 6, that

$$
\begin{aligned}
r^{s_{d}} e^{-\varepsilon n_{1} / n-s_{d}}\left(D\left(K_{1}\right)\right)^{n_{1} / n} & \leq\left(\prod_{j=1}^{d}\left(\operatorname{VDM}\left(\mathcal{L}_{1, j}\right)\right)^{h_{d-j}^{n_{2}}}\right)^{1 / \ell_{d}^{n}} \\
& \leq R^{s_{d}} e^{\varepsilon n_{1} / n-s_{d}}\left(D\left(K_{1}\right)\right)^{n_{1} / n}
\end{aligned}
$$

where $s_{d}:=\left(1 / \ell_{d}^{n}\right) \sum_{j=1}^{d_{0}-1} \ell_{j}^{n_{1}} h_{d-j}^{n_{2}}$ tends to 0 as $d \rightarrow \infty$. We finish the proof by using the same estimate for $K_{2}$ together with Corollary 4 and letting $d$ tend to $\infty$ and then $\varepsilon$ to 0 .

SteP 2. We now prove $(D(K))^{n} \leq\left(D\left(K_{1}\right)\right)^{n_{1}} \cdot\left(D\left(K_{2}\right)\right)^{n_{2}}$. Here, the Leja sequences are of no use but, thanks to $(2)$, elementary considerations on the Chebyshev polynomials will lead to the inequality. Since the graded lexicographic order is compatible with addition, the product of an $\alpha^{1}$-Chebyshev polynomial for $K_{1}$ and an $\alpha^{2}$-Chebyshev polynomial for $K_{2}$ belongs to $\mathbb{U P}_{\left(\alpha^{1}, \alpha^{2}\right)}$ so that $\left.T\left(\left(\alpha^{1}, \alpha^{2}\right)\right), K\right) \leq T\left(\alpha^{1}, K_{1}\right) \cdot T\left(\alpha^{2}, K_{2}\right)$. Hence, writing every $\alpha \in \mathbb{N}_{d}^{n}$ as $\alpha=\left(\alpha^{1}, \alpha^{2}\right)$ with $\alpha^{1}$ (resp. $\alpha^{2}$ ) formed by the first $n_{1}$ (resp. 
last $\left.n_{2}\right)$ coordinates of $\alpha$, we have

$$
\begin{aligned}
& \prod_{|\alpha|=d} T(\alpha, K) \\
& \quad \leq\left(\prod_{j=0}^{d} \prod_{\left|\alpha^{1}\right|=j}\left(T\left(\alpha_{1}, K_{1}\right)\right)^{h_{d-j}^{n_{2}}}\right) \cdot\left(\prod_{j=0}^{d} \prod_{\left|\alpha^{2}\right|=j}\left(T\left(\alpha_{2}, K_{2}\right)\right)^{h_{d-j}^{n_{1}}}\right) .
\end{aligned}
$$

Let $C_{i}>D\left(K_{i}\right)$ for $i=1,2$. In view of $(2)$,

$$
\prod_{\left|\alpha^{i}\right|=d} T\left(\alpha^{i}, K_{i}\right) \leq C_{i}^{d h_{d}^{n_{i}}} \quad \text { for } d \text { large enough, say } d \geq d_{0},
$$

and, for some $R>0$ (depending on the $C_{i}$ 's),

$$
\prod_{\left|\alpha^{i}\right|=d} T\left(\alpha^{i}, K_{i}\right) \leq\left(R C_{i}\right)^{d h_{d}^{n_{i}}} \quad \text { for } d<d_{0}
$$

(both estimates for $i=1,2$ ). Inserting these estimates in (11) (distinguishing the cases $d \geq d_{0}$ and $\left.d \leq d_{0}\right)$ and using Lemma 5 , we find

$$
\left(\prod_{|\alpha|=d} T(\alpha, K)\right)^{1 /\left(d h_{d}^{n}\right)} \leq R^{s_{1, d}+s_{2, d}} \cdot C_{1}^{n_{1} / n} \cdot C_{2}^{n_{2} / n}
$$

where

$$
s_{i, d}=\frac{1}{d h_{d}^{n}} \sum_{j=0}^{d_{0}-1} j h_{j}^{n_{i}} h_{d-j}^{n-n_{i}} \rightarrow 0 \quad \text { as } d \rightarrow \infty .
$$

Now, letting $d \rightarrow \infty$ and using once more (2), we arrive at $D(K) \leq C_{1}^{n_{1} / n}$. $C_{2}^{n_{2} / n}$. The required inequality follows since $C_{i}$ can be taken arbitrarily close to $D\left(K_{i}\right)$. This completes the proof of Theorem 1 .

Acknowledgements. We would like to thank the referee for a careful reading of the manuscript. The research for this note was done in September 2005 when Jean-Paul Calvi was visiting the Department of Mathematics of the Hanoi University of Education. He would like to thank the Hanoi University of Education and the Formath Vietnam program for their support.

\section{References}

[1] T. Bloom, On families of polynomials which approximate the pluricomplex Green function, Indiana Univ. Math. J. 50 (2001), 1545-1566.

[2] T. Bloom and N. Levenberg, Weighted pluripotential theory in $\mathbb{C}^{N}$, Amer. J. Math. 125 (2003), 57-103.

[3] T. Bloom, L. Bos, C. Christensen and N. Levenberg, Polynomial interpolation of holomorphic functions in $\mathbb{C}$ and $\mathbb{C}^{n}$, Rocky Mountain J. Math. 22 (1992), 441-470. 
[4] T. Bloom and J.-P. Calvi, On the multivariate transfinite diameter, Ann. Polon. Math. 72 (1999), 285-305.

[5] - - - Sur le diamètre transfini en plusieurs variables, C. R. Acad. Sci. Paris Sér. I 329 (1999), 567-570.

[6] J.-P. Calvi, Intertwining unisolvent arrays for multivariate Lagrange interpolation, Adv. Comp. Math. 23 (2005), 393-414.

[7] M. Jędrzejowski, Transfinite diameter and extremal points for a compact subset of $\mathbb{C}^{n}$, Univ. Iagell. Acta Math. 29 (1992), 65-70.

[8] M. Schiffer and J. Siciak, Transfinite diameter and analytic continuation of functions of two complex variables, in: Studies in Math. Analysis and Related Topics, Stanford Univ. Press, 1962, 341-358.

[9] V. P. Zaharjuta, Transfinite diameter, Chebyshev constants, and capacity for compacta in $\mathbb{C}^{n}$, Math. USSR-Sb. 25 (1975), 305-364.

Jean-Paul Calvi

Laboratoire Émile Picard (UMR 5580)

Université Paul Sabatier 31062

Toulouse Cedex 9, France

E-mail: calvi@picard.ups-tlse.fr
Phung Van Manh

Department of Mathematics Hanoi University of Education Tuliem, Hanoi, Vietnam E-mail:manhlth@yahoo.com

Received November 8, 2005;

received in final form January 10, 2006 